\title{
Research on 3D Photoelectric Torque Sensor with High Integration
}

\author{
Yuwang Liu ${ }^{1, *}$, Defu Zhang ${ }^{1,2}$ and Fuhua Wang ${ }^{1}$ \\ ${ }^{1}$ Shenyang Institute of Automation Chinese Academy of Science, China \\ ${ }^{2}$ Northeastern University, China \\ *Corresponding author
}

\begin{abstract}
For 3D (three dimensions) photoelectric torque sensor with high Integration, there are two problems. One is the optimization of the layout of multi-measurement units, the other is mismatch of stiffness among the dimensions. According to optimize the layout of multi-measurement units, a scheme is proposed, which is based on the method of dividing into two groups, horizontal and vertical, and differential layout. According to mismatch stiffness among the dimensions, a solution is proposed, which is "turn straight beams into curved". First of all, the layout of multi-measurement units was designed. By means of dividing into two groups, the coupling among the dimensions is reduced, and partial coupling is eliminated by differential layout. At the same time, improve the anti-noise and reduce drift on temperature. Then, elastic element based on cross beam was designed. To solve mismatch of stiffness among the dimensions, cartesian curve replaced straight to design beams. Through finite element analysis, result shows "turn straight beams into curved" can optimize to match stiffness among the dimensions, reduce the maximum stress and improve reliability.
\end{abstract}

Keywords-3D photoelectric torque sensor; the layout of multimeasurement units; mismatch of stiffness among the dimensions

\section{INTRODUCTION}

With the development of photoelectric technology, photoelectric principle has been widely used. Photoelectric torque sensor has also been developed rapidly. Its working principle is the measurement units sense macro deformation of the elastic element by the moment[1]. In 2006, three kinds of elastic element structures were put forward by The University of Tokyo in Japan[2]. They are axial cross beam type, disc spoke type and disc string type. The axial cross beam type can only produce axial torsional deformation, so it only be used in one dimension measurement (Mz). In 2013, the Korea Institute of science and technology developed a 3D torque sensor $(\mathrm{Fz}$, $\mathrm{Mx}, \mathrm{My}$ ), the four measurement units layout with the horizontal and the orthogonal; its disadvantage is the heavy coupling among dimensions[3]. In 2015, the Shenyang Institute of automation chinese academy of sciences proposed a new structure based on flat flexible spring to measure one dimension measurement $(\mathrm{Mz})$. It reduces the phenomenon of stress concentration effectively [4-5]. However, due to the limitation of principle of flat flexible spring, the layout of the measuring units is limited to a large extent, and it is not suitable for the multi-dimensions measurement with multi-measurement units.

At present, research on photoelectric torque sensor is still in the stage of one-dimensional measurement. Multi-dimensional problems: layout of multi-measurement units is single; the structure of elastic element is single; mismatching of stiffness among the dimensions.

Therefore, in this paper, to a flexible joint as the application of the object, a 3D photoelectric torque sensor $(\mathrm{Mz}, \mathrm{Mx}, \mathrm{Fz})$ is developed. The four measuring units are divided into two groups, the horizontal and the vertical. The horizontal group is used for measuring $\mathrm{Mz}$, and the vertical group is used for measuring $\mathrm{Mx}$ and $\mathrm{Fz}$. The $\mathrm{Mz}$ and the $\mathrm{Mx}, \mathrm{Fz}$ are measured to be isolated. Then the two groups are setted the layout of difference and orthogonality to eliminate the partial coupling, improve the anti-noise and reduce drift on temperature[6]. In the design of elastic element, it is designed to meet the minimum requirements based on the cross beam style. For the problem of mismatch of stiffness among the dimensions, using the solution of straight beams into curved makes the match optimize.

\section{WORKING PRINCIPLE}

When be subjected to torque, the elastic element produces a macroscopic deformation. The light blocking plate fixed on it produces the corresponding displacement. Luminous flux of infrared receiver tube changes, so its light flow changes. i.e. The value of current is changed. Then the value of voltage across the $\mathrm{R}_{0}$ is changed. The value of output voltage and the value of torque form one-to-one mapping relationship. In terms of multi-dimensionals measurement, the vector of voltage output and the vector of input torque form one-to-one mapping relationship.

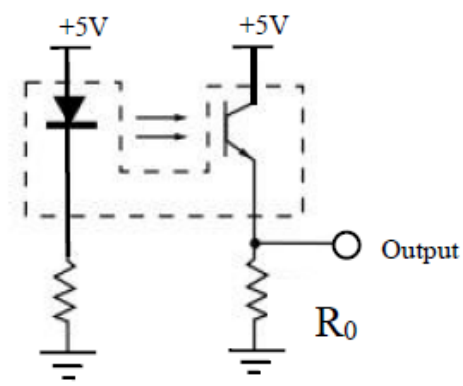

FIGURE I. WORKING CIRCUIT 


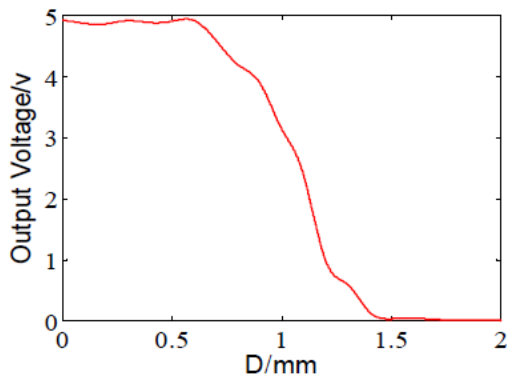

FIGURE II. CHARACTERISTIC CURVE OF VOLTAGE-DISPLACEMENT

\section{LAYOUT PLAN}

There are two main factors that affect the accuracy of the multi-dimensional photoelectric torque sensor: (1) the nonlinearity of the measuring unit. (2) the coupling among dimensions. Therefore, in order to reduce the nonlinearity of the measurement unit and the coupling among dimensions, Multi-measurement units are for reasonable layout.

In practice, the input and output of the measurement unit is not strictly linear. The input of the measuring unit is $\mathrm{x}$, and the output is y. Assuming that the input and output of the measuring unit satisfy the polynomial function relationship:

$$
y=b_{0}+b_{1} x+b_{2} x^{2}+b_{3} x^{3} \cdots+b_{n} x^{n}
$$

When the elastic element is subjected to a moment, if the two measuring units are installed differentially. In that way, an output value is increased, and another output value is reduced:

$$
\begin{aligned}
& y_{1}=b_{0}+b_{1} x+b_{2} x^{2}+b_{3} x^{3} \cdots+b_{n} x^{n} \\
& y_{2}=b_{0}+b_{1}(-x)+b_{2}(-x)^{2}+b_{3}(-x)^{3} \cdots+b_{n}(-x)^{n}
\end{aligned}
$$

(2)-(3), the even items are eliminated:

$$
y=2 b_{1} x+2 b_{3} x^{3} \cdots+2 b_{2 n+1} x^{n+1}, n=0,1,2 \cdots
$$

The differential structure improves the linearity of the measuring unit. At the same time, due to offset the constant $b_{0}$, anti-drift of temperature and anti-noise have also been greatly improved.

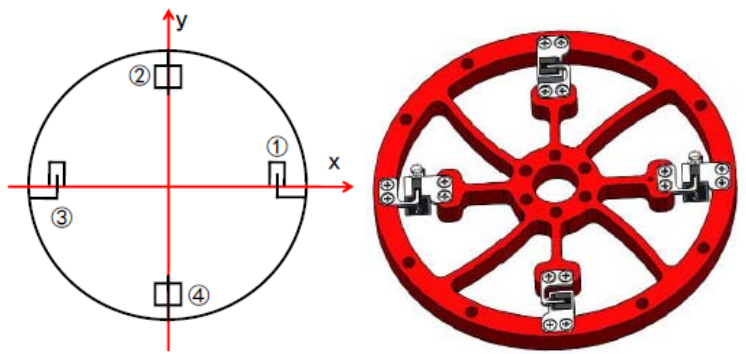

FIGURE III. THE LAYOUT OF MULTI-MEASUREMENT UNITS
As shown in Figure III, the measurement units named (1)(3) form horizontal group, the measurement units named (2)(4) form vertical group. The horizontal group is used for measuring $\mathrm{Mz}$, and the vertical group is used for measuring $\mathrm{Mx}$ and $\mathrm{Fz}$. Set the initial voltages of the measuring units to $\mathrm{U}_{0}$. Set the output voltages of four measuring units when the torque acts to $\mathrm{U}_{\mathrm{P} 1}, \mathrm{U}_{\mathrm{P} 2}, \mathrm{U}_{\mathrm{P} 3}, \mathrm{U}_{\mathrm{P} 4}$. Than the mapping relation between the output voltage vector and the input torque vector is:

$$
\left(\begin{array}{c}
M_{Z} \\
M_{X} \\
F_{Z}
\end{array}\right)=\left[\begin{array}{ccc}
C_{11} & 0 & 0 \\
0 & C_{22} & 0 \\
0 & 0 & C_{33}
\end{array}\right]\left(\begin{array}{c}
\frac{U_{P 1}+U_{P 3}-U_{0}}{2} \\
\frac{U_{P 4}-U_{P 2}}{2} \\
\frac{U_{P 2}+U_{P 4}-U_{0}}{2}
\end{array}\right)
$$

It can be seen from the theory mapping that the two groups are completely isolated in dimensions, which can effectively reduces the coupling among dimensionals.

\section{STRUCTURE OF ELASTIC ELEMENT}

The elastic element is the main part to bear the moment. When bearing the moment, the elastic element not only needs a deformation to match measurement units (deformation requirements as shown in TABLE I), and the stress in a certain value to meet the minimum safety factor. In this paper, the elastic element is made of carbon steel 65Mn, and is designed by the cross type.

TABLE I. RANGE AND DEFORMATION

\begin{tabular}{cccc}
\hline dimension & $\begin{array}{c}\text { range } \\
(\mathbf{n m})\end{array}$ & $\begin{array}{c}\text { the max } \\
\text { deformation(mm) }\end{array}$ & $\begin{array}{c}\text { the best } \\
\text { deformation(mm) }\end{array}$ \\
\hline $\mathbf{M z}$ & $0-3$ & $0.04-0.14$ & 0.08 \\
$\mathbf{M x}$ & $0-3$ & $0.04-0.08$ & 0.65 \\
$\mathbf{F z}$ & $0-100$ & $0.04-0.08$ & 0.65 \\
\hline
\end{tabular}

By adjusting the width and thickness of the rib, the structure can meet the minimal requirements when the width is $2 \mathrm{~mm}$ and the thickness is $4 \mathrm{~mm}$. The figures below show simulational maps of dimensions.
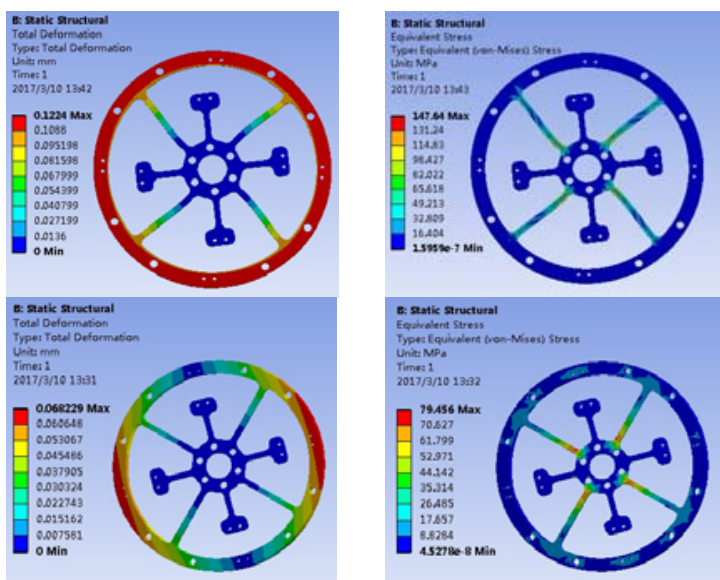

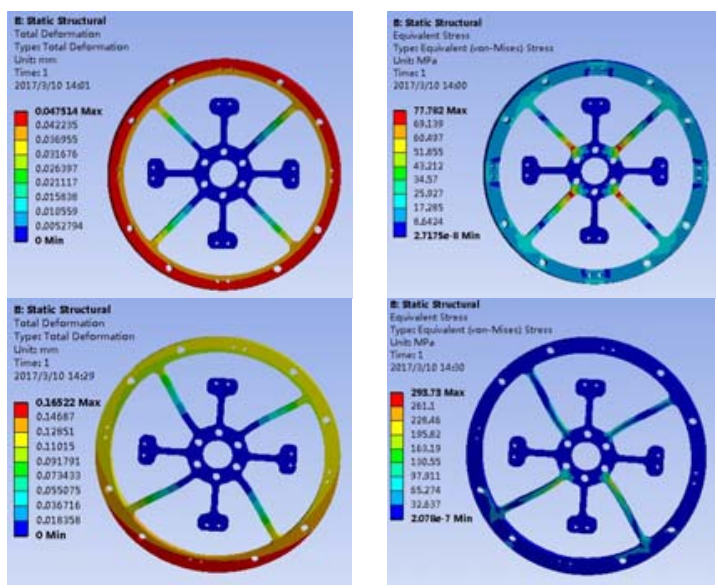

Through the simulation, get the following data, as shown in TABLE II.

TABLE II. SIMULATION DATA OF ELASTIC ELEMENT BY THE CROSS TYPE

\begin{tabular}{ccc}
\hline dimension & $\begin{array}{c}\text { maximum } \\
\text { displacement(mm) }\end{array}$ & $\begin{array}{c}\text { maximum stress } \\
(\mathbf{m m})\end{array}$ \\
\hline $\mathbf{M z}$ & 0.1224 & 0.079215 \\
$\mathbf{M x}$ & 0.068229 & 0.076831 \\
$\mathbf{F z}$ & 0.047514 & 0.051898 \\
all & 0.16522 & 0.14848 \\
\hline
\end{tabular}

As can be seen from the data, although the elastic element meets the minimal requirements, there is a problem of mismatch of stiffness among dimension. The stiffness of the $\mathrm{Mz}$ is small, and the difference between the actual deformation and the optimum deformation is large. Therefore, use the solution "straight beams into curved" to optimize the structure. the curved is the cartesian curve. Its drive equation is:

$$
\left\{\begin{array}{l}
X(t)=\frac{3 \times 35 \tan (t)\left(1+\tan (t)^{2}\right) \cos (t-\pi / 3)}{1+\tan (t)^{3}} \\
Y(t)=\frac{3 \times 35 \tan (t)\left(1+\tan (t)^{2}\right) \sin (t-\pi / 3)}{1+\tan (t)^{3}}
\end{array}(0.05 \leq t \leq 0.32)\right.
$$

The simulation results are obtained:
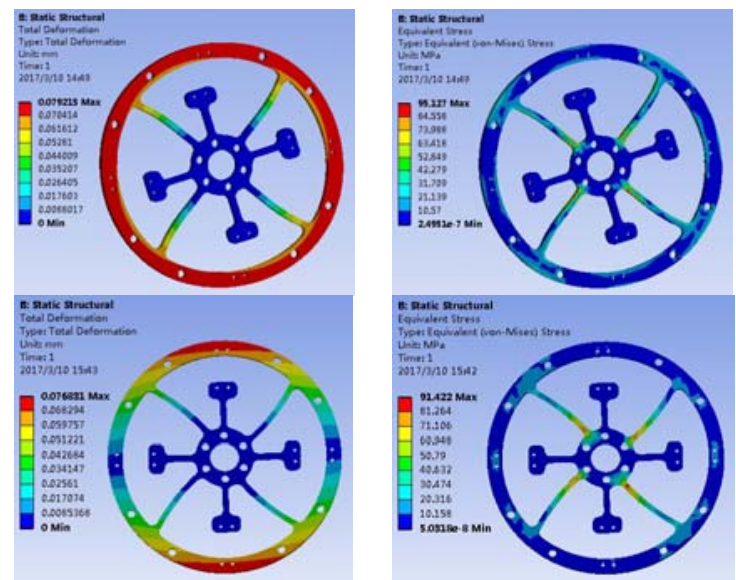
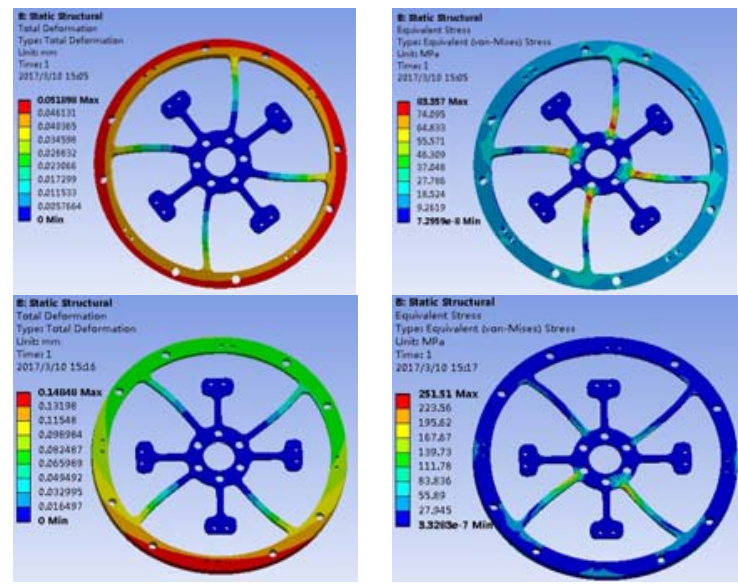

Compare the two structures, as shown in TABLE III.

TABLE III. SIMULATION DATAS OF TWO KINDS OF STRUCTURES

\begin{tabular}{c|c|cc}
\hline \multicolumn{2}{c}{ dimension } & \multicolumn{1}{c}{ cross type } & cross improved \\
\hline $\begin{array}{c}\text { the max } \\
\text { deformation } \\
(\mathbf{m m})\end{array}$ & $\boldsymbol{M}$ & 0.1224 & 0.079215 \\
& $\boldsymbol{F z}$ & 0.068229 & 0.076831 \\
& all & 0.047514 & 0.051898 \\
& $\mathbf{M z}$ & 147.64 & 0.14848 \\
\hline the max stress & $\boldsymbol{M x}$ & 79.456 & 95.127 \\
$\mathbf{( m m )}$ & $\boldsymbol{F z}$ & 77.782 & 81.422 \\
& all & 293.73 & 251.51 \\
\hline \multicolumn{2}{c|}{ safety factor } & 2.689 & 3.141 \\
\hline
\end{tabular}

From the table, In the case of other stiffnesses of dimensionals change slightly, the stiffness of dimension $\mathrm{Mz}$ increases significantly. So match of dimensional stiffness is optimized. At the same time, the maximum stress values decrease, the performance and reliability of the elastic element is improved.

\section{CONCLUSION}

This paper has developed a 3D photoelectric torque sensor. Forward the layout plan of the measurement units which divided into horizontal group and vertical group, orthogonal and differential installation. It reduces the coupling among dimensions and drift of temperature, improves anti-noise. The design of the elastic element structure has optimized the match of dimensional stiffness, improves the performance and reliability of elastic element. It proves the solution "straight beams into curved" is a feasible and effective method to solve the problem of mismatch of dimensional stiffness.

\section{ACKNOWLEDGMENT}

Supported by the National Natural Science Foundation of China (NO: 51605474), Foundation of State Key Laboratory of 
Robotics (NO: 2016-Z09), Natural Science Foundation of Liaoning Province (NO: 20121060)

\section{REFERENCES}

[1] Wilson J S. Sensor Technology Handbook[M]. Singapore: Elsevier Pte Ltd., 2004.

[2] DZMITRY TSETSERUKOU, NAOKI KAWAKAMI, \& SUSUMU TACHI. (2011). Design, control and evaluation of a whole-sensitive robot arm for physical human-robot interaction. International Journal of Humanoid Robotics, 6(04), 699-725.

[3] Kim J C, Kim K S, Kim S. Note: A compact three-axis optical force/torque sensor using photo-interrupters[J]. Review of Scientific Instruments, 2013, 84(12):126109-126109-3.

[4] Liu, Y. W, T. Tian, and J. F. Wu. "A Novel Embedded Torque Sensor of Body Structure Designed and Optimized." 2014 International Conference on Mechanics and Materials Engineering (ICMME 2014) 2014.

[5] Liu YW, Tian T, CHENChen, etal. A Novel Design of Embedded Torque Sensor[J]. 2014.

[6] Gao J, Feng H S, Liu A, et al. Design of New Six-Axis Foot Force Sensor on a Quadruped Robot[J]. Modular Machine Tool \& Automatic Manufacturing Technique, 2010. 